\title{
Successful Treatment of Pulmonary Invasive Aspergillosis with Voriconazole in Patients who Failed Conventional Therapy
}

\author{
J. Garbino, P. Rohner, L. Kolarova, A. Ondrusova, D. Lew
}

\begin{abstract}
Background: The incidence of fungal infections, including those due to Aspergillosis species has continued to increase in recent years. Invasive aspergillosis remains an important cause of morbidity and mortality, despite therapeutics interventions.

Patients and Methods: We reported five cases of invasive pulmonary aspergillosis treated with voriconazole failing to respond to conventional treatments.

Results: The clinical and radiological resolution of pulmonary aspergillosis reported in these cases following therapy with voriconazole is remarkable, considering the infections had proved refractory to standard antifungal therapies. Long-term therapy (in two cases $\geq 1$ year, in one case 6 months) was very well tolerated by patients who were unable to tolerate other antifungal agents.

Conclusion: Therapy with voriconazole offers a new therapeutic option for otherwise difficult-to-treat infections and the potential to significantly improve the management of Aspergillosis infections.
\end{abstract}

Infection 2003; 31: 241-243

DOI 10.1007/s15010-003-3176-4

\section{Introduction}

The incidence of fungal infections, including those due to Aspergillus species, has continued to increase in recent years. Invasive aspergillosis remains an important cause of morbidity and mortality, despite therapeutic interventions [1]. Survival of patients with invasive pulmonary aspergillosis is generally very poor, at least partly due to the lack of effective treatment options for this disease [2]. Aspergillus spp. are widespread in the environment and are the most common cause of invasive mould infections in immunocompromised individuals [3]. Aspergillus infections can result in pneumonia and brain abscesses in this population, which includes patients with burns, HIV infection, cancer, organ transplant recipients and those receiving immunosuppressive therapy [4-6]. Although the overall incidence of invasive aspergillosis is not high, certain patient populations are at particular risk. The disease typically af- fects $5 \%$ of bone marrow transplant recipients, $15 \%$ of patients with hematological malignancies and $10-14 \%$ of solid organ transplantation cases [7,8].

Early initiation of antifungal therapy is associated with improved clinical outcomes [9]. However, as conventional treatments can result in severe adverse reactions and complications, rapid diagnosis of the infection is also vital. The incidence of Aspergillus colonization is very high in respiratory samples, so early diagnosis of invasive pulmonary aspergillosis remains difficult. By the time the infection is confirmed, mortality may reach $50-95 \%$ in patients with disseminated infection [5].

Voriconazole is a new broad-spectrum triazole antifungal agent with activity against a wide range of clinically important and emerging fungal pathogens. Studies have shown voriconazole to result in good clinical responses in $60 \%$ of patients with acute pulmonary or tracheobronchial invasive aspergillosis [10] and to be both more effective and better tolerated than amphotericin B in patients with invasive aspergillosis [11]. When used empirically in febrile neutropenic patients, voriconazole is a suitable alternative to liposomal amphotericin B, with fewer breakthrough infections (including those due to Aspergillus spp.) occurring during therapy with voriconazole compared with amphotericin B [12].

\section{Patients}

\section{Case 1}

A 74-year-old Caucasian male with right nephrectomy due to genitourinary tuberculosis and ischemic cardiopathy since 1998, suffered terminal renal failure due to nephroangiosclerosis. He had received hemodialysis since 1996 and underwent renal transplantation in February 2000. Immunosuppressive therapy consisted of

J. Garbino (corresponding author), L. Kolarova, A. Ondrusova, D. Lew Division of Infectious Disease, Dept. of Internal Medicine, University Hospital Geneva, 24 rue Micheli-du-Crest, $\mathrm{CH}-1211$ Geneva 14, Switzerland; Phone: (+41/22) 372-9839, Fax: -9832, e-mail: jorge.garbino@hcuge.ch P. Rohner

Clinical Microbiology Laboratory, Dept. of Internal Medicine, University Hospital Geneva, Switzerland

Received: August 27, $2002 \bullet$ Revision accepted: December 23, 2002 


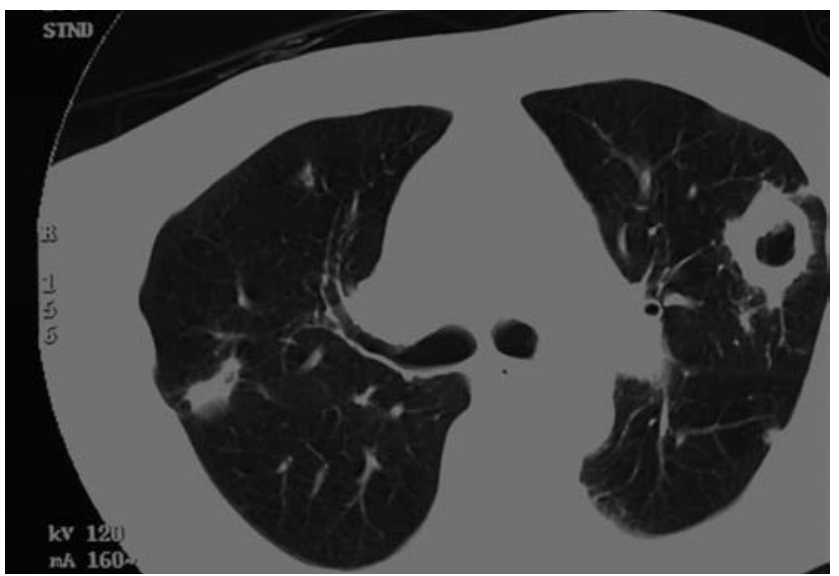

Figure 1. Thoracic CT scan showing a pulmonary rosette-type excavated infiltrate on the left side, bilateral parenchymatous infiltrates and adenopathies.

prednisone (25 mg od), mycophenolate mofetil (1,000 mg bid) and tacrolimus ( $2 \mathrm{mg}$ bid).

Two months after transplantation, the patient was hospitalized due to febrile agranulocytosis in the context of immunosuppressive therapy and pulmonary aspergillosis was suspected on the basis of chest X-rays (cavitary image). Although Aspergillus was not detected in tracheal secretions, the patient was treated with itraconazole (200 $\mathrm{mg}$ bid), due to the high risk of aspergillosis and was discharged from the hospital.

One month later, thoracic CT scans revealed a rosette-type excavated infiltrate on the left side and bilateral parenchymatous infiltrates (Figure 1). Laboratory follow-ups showed an increase in neutrophil count after a further 2 weeks and the patient reported severe asthenia. He was rehospitalized and Aspergillus fumigatus was cultured from bronchoalveolar lavage (BAL); renal insufficiency was still present. Due to the severity of disease, immunosuppressive therapy was reduced and iv voriconazole treatment $(6 \mathrm{mg} / \mathrm{kg} \mathrm{q} 12 \mathrm{~h}$ iv $\times 2$ doses on day 1 , followed by $200 \mathrm{mg}$ bid orally) was initiated. Treatment with amphotericin B was not introduced due to renal impairment. The patient was treated with voriconazole for 1 year, resulting in a clear improvement in thoracic $\mathrm{CT}$ scans (compared to the 1st month) and the pulmonary lesions were considered cured.

Adverse events observed during voriconazole treatment were dizziness and ocular disturbances, which both occurred during the initial infusion, a few minutes later the patient recovered without sequelae. Voriconazole treatment was well tolerated for 1 year and no liver function test abnormalities were observed.

\section{Case 2}

A 37-year-old Caucasian male with a past medical history of chronic granulomatous disease since the age of 14 years, inguinal adenitis caused by Candia albicans, pulmonary anguilliasis and type 1 diabetes mellitus presented in January 2000 with tracheobronchitis and received clarithromycin therapy.

One month later, the patient reported recurrence of cough with purulent sputum, night sweating and weight loss (3-4 kg). A subcutaneous tumefaction was noted below the left breast and $A$. fumigatus was cultured from the biopsy. Thoracic CT scans indi-

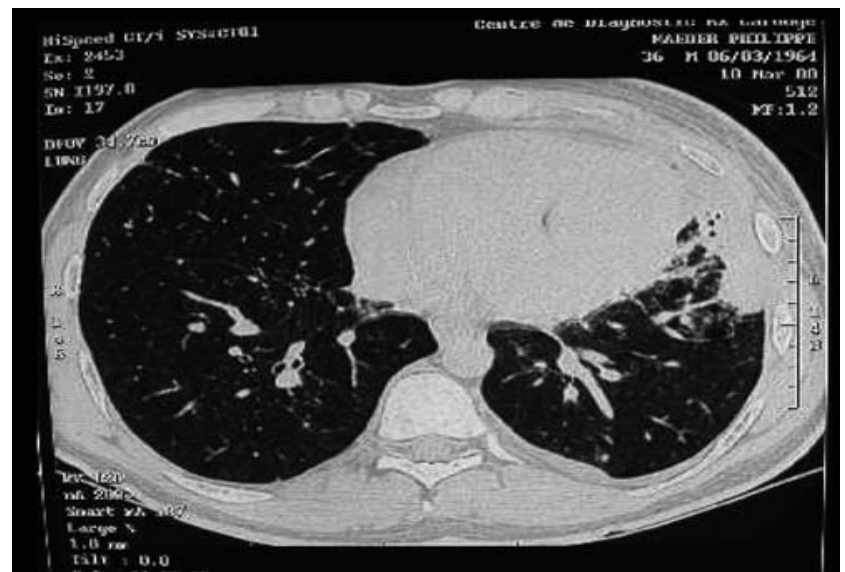

Figure 2. Thoracic CT scan showing a pulmonary abscess in contact with the pericardium and chest wall, mediastinal adenopathies, infiltrates and pleuro-parenchymatous synechia in the lingual lobe.

cated a large subpectoral mass on the left side, corresponding with an area of pleural thickness. The scans also revealed osteolytic changes to the fourth and fifth anterior left ribs, a pulmonary abscess in contact with pericardium, mediastinal adenopathies, infiltrates and pleuro-parenchymatous synechia in the lingula lobe (Figure 2). Bone scintigraphy showed hyperactivity in the fourth and fifth anterior left ribs.

Treatment was started with conventional amphotericin B ( $1 \mathrm{mg} / \mathrm{kg} /$ day), but was switched to a lipid formulation 11 days later (Ambisome ${ }^{\circledR} 3 \mathrm{mg} / \mathrm{kg} / \mathrm{day}$ ) due to adverse events (anemia and renal insufficiency). This treatment regimen was maintained for 6 weeks, during which time renal insufficiency improved, but a blood transfusion was necessary to correct the persistent anemia. The patient was then switched to therapy with voriconazole $(6 \mathrm{mg} / \mathrm{kg}$ $\mathrm{q} 12 \mathrm{~h}$ iv $\times 2$ doses on day 1 , followed by $200 \mathrm{mg}$ bid orally). One month later, CT scans indicated that the pectoral mass on the left side had resolved and that the infiltrates in the superior left lobe were slightly reduced. A small parenchymatous pulmonary condensation at the level of the lingual lobe persisted, as did the adenopathies. A follow-up CT scan 1 year later showed further improvement in the pulmonary infiltrates.

The patient tolerated voriconazole treatment very well. After 1 year of treatment, a cutaneous rash attributable to the drug occurred, which improved when voriconazole was reduced from $200 \mathrm{mg}$ bid to $150 \mathrm{mg}$ bid orally. The patient continues to receive prophylactic treatment with voriconazole.

\section{Case 3}

A 65-year-old Caucasian male had a past medical history of viral pericarditis and idiopathic pulmonary fibrosis, which was treated with immunosuppressive drugs.

In November 1998, the patient underwent bi-pulmonary transplantation. Three days later, A. fumigatus and C. albicans were cultured from the respiratory tract. In the context of immunosuppressive therapy and suspected pulmonary aspergillosis with a compatible chest X-ray, excavated infiltrates, itraconazole therapy was initiated (400 mg/day). Bronchoscopy 1 week later revealed sutures covered by plaques on the left side, which reached the right superior bronchus and biopsy cultured A. fumigatus. Amphotericin $\mathrm{B}$ was stopped due to patient's intolerance. Due to lack of response 
to itraconazole, voriconazole therapy was started 1 month after transplantation $(6 \mathrm{mg} / \mathrm{kg} \mathrm{q} 12 \mathrm{~h}$ iv $\times 2$ doses on day 1 , followed by $200 \mathrm{mg}$ bid orally). Respiratory tract cultures became negative and the infection was considered cured after 3 months of treatment. The patient died 5 months later, due to a cardiorespiratory arrest.

\section{Case 4}

A 56-year-old Caucasian male had a past medical history of a leukemia diagnosed in 2001 and wastreated with chemotherapy. During chemotherapy, the patient developed thrombocytopenia and severe anemia, which necessitated blood transfusions. Neutropenia below 500 granulocytes/1 lasted for 2 days.

In September 2001, blepharoconjunctivitis occurred and the patient was treated with cefepime and teicoplanin for 4 weeks; acyclovir was also administered for 3 weeks, due to positive herpes serology. One month later, the patient became febrile and a fungal infection was suspected. Amphotericin B therapy $(1 \mathrm{mg} / \mathrm{kg} /$ day $)$ was initiated, and pulmonary aspergillosis was subsequently confirmed by thoracic CT scans (halo sign) and positive serologic test (galactomanan). Due to intolerance of the amphotericin B treatment (renal impairment and hypokalemia), the patient was switched to voriconazole $(6 \mathrm{mg} / \mathrm{kg} \mathrm{q} 12 \mathrm{~h}$ iv $\times 2$ doses on day 1 , followed by $200 \mathrm{mg}$ bid orally) for 6 months, which resulted in resolution of the radiological images. The patient did not present any drug intolerance.

\section{Case 5}

A 77-year-old Caucasian male had a past medical history of respiratory insufficiency due to pulmonary fibrosis and was treated with prednisone $(20 \mathrm{mg} /$ day $)$ and azathioprine $(25 \mathrm{mg} /$ day $)$.

The patient was admitted to hospital with bronchopneumonia and respiratory insufficiency. Antimicrobial treatment (imipenem, clarithromycin) was initiated, but no clinical response was observed. Invasive pulmonary aspergillosis was diagnosed by lung biopsy (cultures grew $A$. fumigatus), and itraconazole therapy $(400 \mathrm{mg} /$ day) was started. After 1 week, no clinical improvement was observed and treatment was switched to amphotericin B $(1 \mathrm{mg} / \mathrm{kg} /$ day $)$. The patient developed renal failure and was switched to voriconazole $(6 \mathrm{mg} / \mathrm{kg}$ q12 $\mathrm{h}$ iv $\times 2$ doses on day 1 , followed by $200 \mathrm{mg}$ bid orally). The patient's clinical condition worsened, he developed septic shock and was transferred to the intensive care unit. He required mechanical ventilation and vasopressors (ephedrine and dobutamine), but died 3 days later; death was not attributable to fungal infection.

\section{Discussion}

The clinical and radiological resolution of pulmonary aspergillosis reported in these cases following therapy with voriconazole is remarkable, considering the infections had proved refractory to standard antifungal therapies. Longterm therapy (in two cases $\geq 1$ year, in one case 6 months) was very well tolerated by patients who were unable to tolerate other antifungal agents, including those with underlying conditions such as renal insufficiency, which can restrict the selection of conventional antifungal agents. Voriconazole appears to offer a new therapeutic option for otherwise difficult-to-treat infections, such as those in patients with chronic granulomatous disease. Echinocandins could be another treatment alternative. Therapy with voriconazole also offers the potential to significantly improve the management of Aspergillus infections. In the cases reported here, aspergillosis was considerably improved or resolved and the clinical conditions of these individuals were stabilized. Using voriconazole to stabilize patients with previously difficult-to-treat infections may ultimately allow long-term therapeutic interventions, such as bone marrow transplantation, to be undertaken.

\section{Acknowledgment}

Voriconazole was provided by Pfizer Inc. in the context of compassionate use treatment.

\section{References}

1. Kontoyiannis DP, Bodey GP: Invasive aspergillosis in 2002: an update. Eur J Clin Microbiol Infect Dis 2002; 21: 161-172.

2. Oren I, Goldstein N: Invasive pulmonary aspergillosis. Curr Opin Pulm Med 2002; 8: 195-200.

3. Warnock DW, Hajjeh RA, Lasker BA: Epidemiology and prevention of invasive aspergillosis. Curr Infect Dis Rep 2001; 3 507-516.

4. McKinsey DS, Wheat LJ, Cloud GA, et al.: Itraconazole prophylaxis for fungal infections in patients with advanced human immunodeficiency virus infection: randomized, placebo-controlled, double-blind study. National Institute of Allergy and Infectious Diseases Mycoses Study Group. Clin Infect Dis 1999; 28: 1049-1056.

5. Paterson DL, Singh N: Invasive aspergillosis in transplant recipients. Medicine (Baltimore) 1999; 78: 123-138.

6. Vogeser M, Wanders A, Haas A, Ruckdeschel G: A four-year review of fatal aspergillosis. Eur J Clin Microbiol Infect Dis 1999 18: 42-45.

7. Denning DW, Evans EG, Kibbler CC, et al.: Guidelines for the investigation of invasive fungal infections in haematological malignancy and solid organ transplantation. British Society for Medical Mycology. Eur J Clin Microbiol Infect Dis 1997; 16: 424-436.

8. Morrison VA, Haake RJ, Weisdorf DJ: The spectrum of non-Candida fungal infections following bone marrow transplantation Medicine (Baltimore) 1993; 72: 78-89.

9. Ellis $M$ : Invasive fungal infections: evolving challenges for diagnosis and therapeutics. Mol Immunol 2002; 38: 947-957.

10. Denning DW, Ribaud P, Milpied N, et al.: Efficacy and safety of voriconazole in the treatment of acute invasive aspergillosis. Clin Infect Dis 2002; 34: 563-571.

11. Herbrecht R, Denning D, Patterson T, et al.: Voriconazole versus amphotericin $\mathrm{B}$ for primary therapy of invasive aspergillosis. N Engl J Med 2002; 347: 408-415.

12. Walsh TJ, Pappas P, Winston DJ, et al.: Voriconazole compared with liposomal amphotericin B for empirical antifungal therapy in patients with neutropenia and persistent fever. N Engl J Med 2002; 346: 225-234. 